\title{
Critical N-Acetylcysteine Treatment Evaluation with Acetaminophen Overdose Management, A Case Report
}

Dr. Ahmed O Alenazi. B.Sc., Pharm D, CACP ${ }^{1,3,4}$, Dr. Junaid Alam DABIM ${ }^{2,3,4}$, Dr. Juned Loharia. J MD, MRCP (UK), $\mathrm{EDIC}^{2,3,4}$, Dr. Abdulwaheed Shaikh MBBS, MCPS (Pulmonology) ${ }^{2,3,4}$

${ }^{1}$ Pharmaceutical Care Department, Imam Abdulrahman Bin Faisal Hospital, Ministry of the National Guard, Dammam, Saudi Arabia;

${ }^{2}$ Department of Medicine, Imam Abdulrahman Bin Faisal Hospital, Ministry of the National Guard, Dammam, Saudi Arabia;

${ }^{3}$ King Abdullah International Medical Research Center, Al Ahsa, Saudi Arabia;

${ }^{4}$ King Saud bin Abdul-Aziz University for Health Sciences, Al Ahsa, Saudi Arabia

DOI: $\frac{10.36348 / \text { sjmps.2020.v06i05.002 }}{\text { | Received: 29.04.2020 | Accepted: } 07.05 .2020 \text { | Published: } 16.05 .2020}$

Abstract

Acetaminophen is the most prevalent, deliberately ingested medication in overdose episodes and a key cause of acute liver failure. N-Acetylcysteine (NAC) is used to treat an overdose of acetaminophen. Using NAC may cause rare fatal adverse events which could affect the safety of the patient. Generally, the type and frequency of NAC related events are too minimal to be considered as an adverse reaction. It is necessary to examine the route of administration, method of reaction detection and the nature of the reactions. These events does not preclude patients from receiving this hepatoprotective advantage, particularly in the context of significant acetaminophen ingestion. We present our case and highlight the clinical inference which can be derived from this entity.

Keywords: Acetaminophen, Anaphylaxis, Hepatotoxicity, N Acetyl cysteine.

Copyright @ 2020: This is an open-access article distributed under the terms of the Creative Commons Attribution license which permits unrestricted use, distribution, and reproduction in any medium for non-commercial use (NonCommercial, or CC-BY-NC) provided the original author and source are credited.

\section{INTRODUCTION}

Acetaminophen (paracetamol) has been used as an analgesic and antipyretic since the mid-20th century, after being discovered in Germany at the end of $19^{\text {th }}$ century [1]. It is an over-the-counter (OTC) drug globally. The recommended maximum daily dose is 4 gram [6] with a peak plasma level reached within an hour after oral ingestion. In an overdose, peak levels may only be reached after 4 hours. The liver metabolism occurs through conjugation with glucoronide and sulfate or oxidation by cytochrome P450. The product of this metabolic process is N-acetylp-benzoquinoneimine (NAPQI) which binds to glutathione in hepatocytes forming a nontoxic metabolite [8].

In the last seven decades, acetaminophen overdose has remained a frequent cause of emergency hospital admissions with the majority of the patient's female, with a median age of 30 years, and attempting to commit suicide $[2,3]$. The first hepatotoxicity report in humans due to acetaminophen toxicity was published in 1966[1]. Globally, acetaminophen is used in 30-40\% of the intentional drug overdose [2,4]. Recent studies from Saudi Arabia also indicated that acetaminophen overdose is the cause of $30 \%$ of deliberate medication overdose, with the majority female and $40 \%$ of the patients had an history of alcohol use and/or psychiatric disorder [2, 3].

The hepatotoxic effect of acetaminophen is dose dependent with evidence that a high dose of 150 mg per kilogram or 10-15 gram (20-30 tablets of 500 milligram) causing acute liver failure[4]. This hepatocellular injury occurs due to an interaction of extra NAPQI metabolites with the protein and nucleic acids of the hepatocytes ${ }^{5}$. Malnutrition and alcohol consumption as additional risk factors increase the risk of hepatotoxicity, even with regular acetaminophen doses [2-4].

$\mathrm{N}$-acetyl cysteine (NAC) is a precursor for glutathione synthesis. Used as an antidote, it binds to the acetaminophen metabolite NAPQI in the hepatocytes and prevent liver damage[4], when given within 8-10 hours from the overdose ingestion ${ }^{6}$. The route of NAC administration is intravenous or oral, and over a varying period of 21 to 72 hours as described in literature. Different protocols are applied to manage acetaminophen overdose (Table 1) 
Table-1: N-acetyl cysteine (NAC) Protocols Intravenous (IV) protocol over 21 hours:

- $\quad$ Loading dose of $150 \mathrm{mg} / \mathrm{kg}$ over one hour

- $\quad$ Then $50 \mathrm{mg} / \mathrm{kg}$ over 4 hours

- Then finally with $100 \mathrm{mg} / \mathrm{kg}$ over 16 hours

Oral protocol over 72 hours:

- $\quad$ Loading dose $140 \mathrm{mg} / \mathrm{kg}$

- $\quad$ Then $70 \mathrm{mg} / \mathrm{kg}$ every 4 hours for an additional 17 doses.

There is no difference in the efficacy of the IV and oral protocols. The IV protocol is preferred because of the short management duration and fewer gastrointestinal adverse effects. In the case of a rare chance of a dose miscalculation, an anaphylactic reaction may occurred. The oral dose is usually mixed with a soft drink or juice to enhance the taste tolerability [6] with the most common adverse effect nausea and vomiting.

We describe a case where side effects altered the course of management and subsequent clinical implications.

\section{Case Report}

A 30-year old Saudi female nurse, single, living alone with background history of bronchial asthma with inhalers prescribed, depression not currently on any follow-up or medical treatment and a history of sleeve gastric surgery 5 years ago. She was brought to the emergency by her friend with multiple episodes of vomiting for a few hours after the deliberate ingestion of 20 tablets of combination drug, which contain acetaminophen $300 \mathrm{mg}$ and Chlorzoxazone 250 $\mathrm{mg}$ in each tablet. She had no history of any other drug abuse. She gave a history of occasional alcohol intake in addition to social isolation and manifested symptoms of suicidal ideation. She was vitally stable on the initial assessment, conscious, oriented, with a soft abdomen as well as normal liver, renal and hematological parameters. Emergency management was provided with an anti-emetic, fluids and the acetaminophen level was requested. Awaiting the levels, she was admitted for further management and close monitoring of her liver, coagulation and clinical parameters. In view of the unavailability of the levels immediately at the point of care and the ingestion history of a significant dosage, a decision was taken to initiate antidote management with NAC according to the standard 21-hour protocol. The patient received the first hour loading dose and before it was completed, she had itching with no rash, signs of mild angioedema including swelling of the face and lips, no breathing difficulty or wheezes and no hemodynamic instability. Her symptoms improved after receiving hydrocortisone and diphenhydramine. We consulted an immunologist who advised us to continue the NAC at a reduced fluid rate to lengthen the total administration duration as well as maintaining the intravenous steroids, montelukast and antihistamine. Her symptoms resolved and we followed the patient according to the Rumack-Matthew nomogram and completed the NAC treatment without any further complications. When the levels were received from the external laboratory, the initial acetaminophen concentration was 12.2 microgram per milliliter followed by 1.6 microgram per milliliter after 4 hours. Her follow-up liver function tests remained normal. She was assessed by a psychiatrist and diagnosed with a major depressive disorder with a bipolar affective disorder. Medication was prescribed as well as regular outpatient follow-up consultations. On subsequent follow-up visits, she was maintained on her regular treatment for depression with no relapse of any deliberate self-harming behavior.

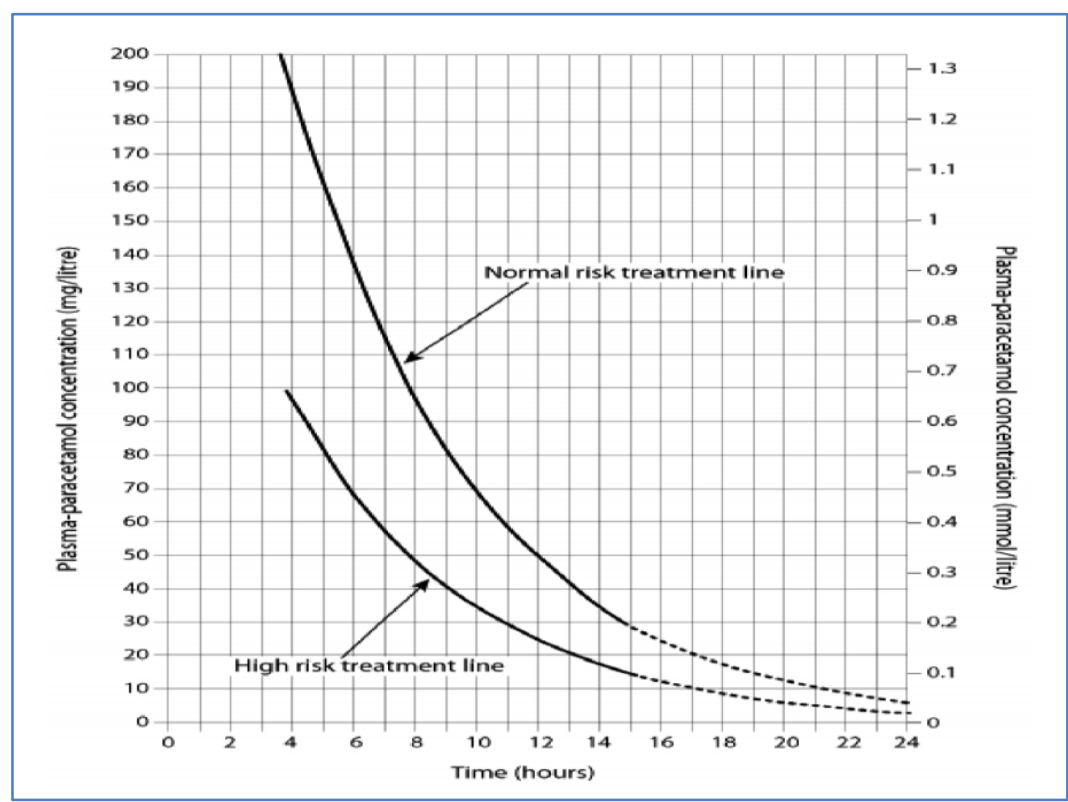

Fig-1: Rumack Matthew nomogram [6] 


\section{DiscuSSION}

An acetaminophen overdose is one of the most frequent causes of medication poisoning and death. Liver injury may occur after a single acute ingestion of supra-therapeutic or even multi ingestion of therapeutic amounts. Acetaminophen overdose symptoms may not be detected in a patient presenting within 24 hours. Some of these symptoms, such as nausea, vomiting, abdominal pain, jaundice, renal injury, coagulopathy, and encephalopathy may present later. The severity following an acute ingestion is quantified by plotting a timed serum acetaminophen concentration on the modified Rumack-Matthew nomogram (Figure 1). The duration of the NAC management depends on the serum alanine aminotransferase (ALT) concentrations.

NAC is strongly indicated when the serum acetaminophen concentration at 4 hours or more following acute ingestion of an immediate-release preparation is above the treatment line of the treatment nomogram for acetaminophen poisoning. NAC is also indicated in a suspected single ingestion of greater than $150 \mathrm{mg} / \mathrm{kg}$ (7.5 grams total dose regardless of weight) in a patient whose serum acetaminophen concentration will not be available for more than 8 hours from the time of ingestion[7], a patient with an unknown ingestion time and a serum acetaminophen concentration $>10 \mathrm{mcg} / \mathrm{mL}$ (66micromol/L), a patient with a history of acetaminophen ingestion and any evidence of liver Injury, a patient with a delayed presentation ( $>24$ hours after ingestion) consisting of laboratory evidence of liver injury (ranging from mildly elevated aminotransferases to fulminant hepatic failure) and a history of excessive acetaminophen ingestion.

Non-allergic anaphylactic reactions (NAAR) during treatment with IV NAC can be reduced by using a two-bag regimen instead of the traditional three-bag regimen in 20 hours protocol. In a retrospective study, NAAR occurred in $10 \%$ of 389 patients treated with the standard regimen versus $4.3 \%$ of 210 patients treated with a modified two-bag regimen [8]. There are no comparative trials comparing the 20-hour IV and the 72-hour oral treatment protocols. The best available data suggest that both routes are effective and the differences are minimal [9]. The IV administration is favored for patients with vomiting or other contraindications to an oral administration (i.e., pancreatitis, bowel ileus or obstruction, bowel injury) or hepatic failure.

In terms of allergic reactions to NAC, the clinical presentation vary. There are no clear guidelines available regarding the continuation of IV NAC in patients with anaphylaxis. However, it is standard that a patient, experiencing flushing without pruritus or urticarial, do not require an intervention and the infusion can be continued, However, in patients who develop urticaria, the infusion should be stopped and treatment with intramuscular epinephrine, diphenhydramine and glucocorticoid initiated. The infusion can be restarted at the prior rate once the urticaria resolves. For patients with angioedema or respiratory symptoms, the infusion should be stopped, the patient treated with epinephrine, diphenhydramine, glucocorticoid and, if wheezing, salbutamol. In this group of patients, when the signs and symptoms resolve, the infusion may be restarted at the prior rate an hour after the administration of epinephrine. Lastly, in patients who develop hypotension or other persistent systemic anaphylaxis symptoms after the IV NAC therapy, the infusion is terminated, treatment for anaphylaxis is provided and the IV NAC should not be restarted [10].

Our patient manifested with pruritus and angioedema, but no wheezing, and was treated with steroids and antihistamine. She did not receive epinephrine and once the acute manifestations improved, the NAC therapy was restarted at a slower infusion rate and she completed the treatment uneventfully.

\section{Conclusion}

The majority of acetaminophen overdose patients treated with NAC do not manifest any safety issues. NAC is both safe and efficacious. However, in some instances, such as this case illustrates, the treatment requires awareness with a careful risk/benefit assessment, precise dose calculation and a slow drug infusion rate with the immediate availability of resuscitation equipment.

\section{REFERENCES}

1. Sheen, C. L., Dillon, J. F., Bateman, D. N., Simpson, K. J., \& Macdonald, T. M. (2002). Paracetamol toxicity: epidemiology, prevention and costs to the health- care system. Qjm, 95(9), 609-619.

2. Al Suliman, H. R., Al-Mowallad, S. M., Jadou, N. S., Al Somali, A. S., Almokhlef, S. A., Alghamdi, W. A., ... \& Alabdulwahab, M. M. (2019). Analysis of paracetamol overdose samples in saudi arabia. Indo american journal of pharmaceutical sciences, 6(1), 2327-2331.

3. Almansori, M. A., Alhammadi, H. I., \& Almulhim, F. A. (2015). Paracetamol overdose: Analysis of a sample from a tertiary hospital in Eastern Saudi Arabia. Saudi Journal of Medicine and Medical Sciences, 3(3), 209.

4. Bateman, D.N.(2007). Poisoning: Focus on paracetamol. J R Coll Physicians Edinb, 37(4):3324.

5. Lee, W. M. (2003). Drug-induced hepatotoxicity. New England Journal of Medicine, 349(5), 474-485. 
6. Au, V., \& Zakaria, M. I. (2014). A study on the medication errors in the administration of $\mathrm{N}$ acetylcysteine for paracetamol overdose patients in Malaysia.Hong Kong Journal of Emergency Medicine, 21(6), 361-367.

7. Prescott, L. F., Illingworth, R. N., Critchley, J. A., Stewart, M. J., Adam, R. D., \& Proudfoot, A. T. (1979). Intravenous $\mathrm{N}$-acetylcystine: the treatment of choice for paracetamol poisoning. $\mathrm{Br} \mathrm{Med}$ J, 2(6198), 1097-1100.

8. Wong, A., \& Graudins, A. (2016). Simplification of the standard three-bag intravenous acetylcysteine regimen for paracetamol poisoning results in a lower incidence of adverse drug reactions. Clinical Toxicology, 54(2), 115-119.

9. Green, J. L., Heard, K. J., Reynolds, K. M., \& Albert, D. (2013). Oral and intravenous acetylcysteine for treatment of acetaminophen toxicity: a systematic review and metaanalysis. Western Journal of Emergency Medicine, 14(3), 218.

10. Bailey, B., \& McGuigan, M. A. (1998). Management of anaphylactoid reactions to intravenous $\mathrm{N}$-acetylcysteine. Annals of emergency medicine, 31(6), 710-715. 